\title{
VISUDYNE® (verteporfin) ABBREVIATED PRESCRIBING INFORMATION
}

Presentation: Glass vial containing $15 \mathrm{mg}$ of verteporfin as powder. Indications: Treatment of age-related macular degeneration in patients with predominantly classic subfoveal choroidal neovascularisation or subfoveal choroidal neovascularisation secondary to pathological myopia. Dosage and administration: A ten minute intravenous infusion of Visudyne ( $30 \mathrm{ml}$ solution) at a dose of $6 \mathrm{mg} / \mathrm{m}^{2}$ body surface area. This is followed by the activation of Visudyne 15 minutes after the start of the infusion using a diode laser generating non-thermal red light (wavelength $689 \mathrm{~nm}( \pm 3 \mathrm{~nm})$. At the recommended light intensity of $600 \mathrm{~mW} / \mathrm{cm}^{2}$, it takes 83 seconds to deliver the required light dose of $50 \mathrm{~J} / \mathrm{cm}^{2}$. Reevaluate every 3 months; if recurrent CNV leakage occurs, Visudyne therapy may be given up to 4 times per year. Contraindications: Porphyria, known hypersensitivity to verteporfin or to any of the excipients, or severe hepatic impairment. Precautions: Due to photosensitivity, avoid exposure of unprotected skin, eyes or other body organs to direct sunlight or bright indoor light for 48 hours after infusion. UV sunscreens are not effective at protecting against photosensitivity reactions. Exercise caution in moderate hepatic impairment, biliary obstruction, unstable heart disease, uncontrolled arterial hypertension and treatment under general anaesthesia. If severe decrease of vision (equivalent to 4 lines or more) occurs within 1 week after treatment, do not re-treat at least until vision completely recovers to pretreatment level. If extravasation occurs, stop infusion immediately. Protect the affected area thoroughly from bright direct light until swelling and discolouration have disappeared. Patients should be under close medical supervision during Visudyne infusion. Chest pain, vasovagal reactions (posture related) and hypersensitivity reactions have been reported. Interactions: No specific drug-drug interaction studies have been conducted in humans. Concomitant use of other photosensitising agents (e.g. tetracyclines, sulphonamides, phenothiazines, sulphonylurea, hypoglycaemic agents, thiazide diuretics, and griseofulvin) could increase the potential for photosensitivity reactions. Pregnancy and lactation: Visudyne should be used in pregnant women only if the benefit justifies the potential risk to the foetus. Do not administer to nursing mothers or stop breastfeeding for 48 hours after administration. Effects on ability to drive and use machines: Do not drive or use machines as long as symptoms such as abnormal vision persist. Undesirable effects: Ocular: Common (1-10\%): Abnormal vision such as blurry, hazy, fuzzy vision, or flashes of light, decreased vision, visual field defects such as grey or dark haloes, scotoma and black spots, severe but usually transient vision reduction. Uncommon (0.1-1\%): retinal detachment (non-rhegmatogenous), subretinal haemorrhage, vitreous haemorrhage. Rare $(<0.1 \%)$ : retinal or choroidal vessel non-perfusion. Injection site side effects: Common (1-10\%): Pain, oedema, extravasation, inflammation. Uncommon (0.1-1\%): haemorrhage, hypersensitivity, discolouration. Systemic side effects: Common (1-10\%): Nausea, photosensitivity reaction, back pain, pain in the pelvis, shoulder girdle or rib cage during infusion, asthenia, pruritus, hypercholesterolaemia. Uncommon (0.1-1\%): Pain, hypertension, hypesthesia, fever. Rare $(<0.1 \%)$ : chest pain, vasovagal reactions (posture related), hypersensitivity (syncope, sweating, dizziness, rash, dyspnoea, flushing, changes in blood pressure and heart rate).

Adverse events should be reported. Reporting forms and information can be found at www.yellowcard.gov.uk. Adverse events should also be reported to Novartis Pharmaceuticals UK Ltd on (01276) 698370.

Legal category: POM. Packaging quantities: Each vial containing $15 \mathrm{mg}$ verteporfin. Price: UK £850. Marketing authorisation number: EU/1/00/140/001.

Marketing authorisation holder: Novartis Europharm Limited, Wimblehurst Road, Horsham, West Sussex, RH12 5AB, United Kingdom. Date of preparation: $24^{\text {th }}$ October 2008. Visudyne is a registered Trade Mark. Full prescribing information, including SmPC, is available from: Novartis Pharmaceuticals, Frimley Business Park, Frimley, Camberley, Surrey, GU16 7SR. Telephone: 01276 692255. Fax: 01276692508. 